\title{
Building renovations in BIM systems
}

\author{
Marian Smutný ${ }^{*}$ \\ ${ }^{1}$ CTU Prague, Faculty of Civil Engineering, Thákurova 7, 16629 Praha 6, Czech Republic
}

\begin{abstract}
Renovation of buildings is a demanding challenge for computer systems. Nowadays, complex 3D models of historic buildings can be scanned and modelled, including sculptural filigrees and stucco decorations with possibility to generate different projections, cross-sections and elevations according to descriptive geometry rules. However, all of this falls within the field of "3D Modelling". BIM systems work with elements that have to be classified according to their purpose, or according to the way they are realized. The system is then able to sort, filter or group them. Also, it is able to compute their bill of quantity and quality based on their attributes. Last but not least, it has to be able to schematically display civil engineering drawings on the basis of classification of the elements which differ considerably from the display of descriptive geometry. In regular constructions, repeatability and predictability of the links between the elements suit computer systems. Thus, the issue of building renovations in BIM systems is to find a balance between modelling irregular constructions and irregular space arrangements, while maintaining the ability to interact with other BIM elements.
\end{abstract}

\section{Introduction}

BIM programs are based on 3 different engines:

1. object-oriented $(1,2)$ engine - each object serves a separate function. It is defined by its properties' - what it is and what it can do. Examples of such BIM programs are ArchiCAD, VectorWorks.

2. database $(3,4)$ engine - all information is stored and maintained in a relation database from which visual representation of drawings and models are generated. Examples of such BIM programs are Speedikon, Revit.

3. geometry modeller (5) engine - all information is stored as a 3D model with attached attributes. It may be based on a parametric modeller as Parasolid or ACIS, or one can use a specialised modeller. Examples of such BIM programs are AutoCAD Architectural, TriForma, and Allplan.

Each of these approaches gives certain advantages and certain disadvantages. In this article the Allplan BIM system is described - how it is able to create a virtual building with

\footnotetext{
* Corresponding author: marian.smutny@fss.cvut.cz
} 
irregular constructions (walls, rooms, openings and vaults) of historic buildings and their modifications while keeping their interactivity with reasonable accuracy and high returns.

\section{The Modeller}

Allplan BIM modeller is not based on a robust parametric modeller but on a specific simplified modeller which is particularly suited for building renovations mainly because of two features:

Modeller is based on simple 3D solids consisting of a polygonal plan shape extruded with top and bottom faces of a spatial envelope (in Allplan terminology the term "Planes", or "Roof Planes" is used) which constitutes a storey. Top and bottom shapes of 3D solids can be referenced to top or bottom "Planes" in various distances, or can be fixed independently from "Planes". The advantage of this approach is that the architect can focus on the floor plan, and the modeller ensures that constructions will fit to the storey height especially when it is formed by vaults.

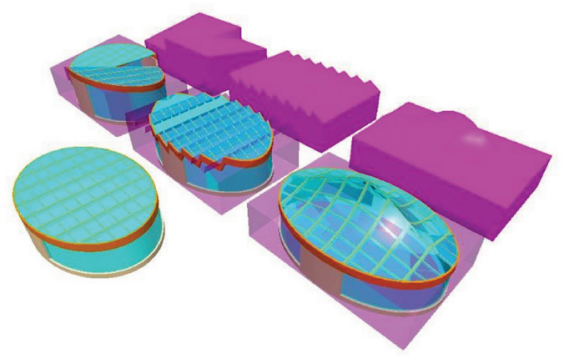

Fig. 1. Violet colour represents a virtual spatial envelope "Roof Planes" where BIM elements continuously adapt to each of them.

The modeller uses both positive and negative 3D solids. Positive 3D solids are the basis for walls, beams, slabs, etc. Negative 3D solids are the basis for openings such as doorways, windows, niches, etc. The big advantage with the renovation of buildings is it can be placed independently or can be overlapped. Thus, the window can be traversed through a folded wall or wall connection. Equally, it is possible to freely add and remove a plan vertex to floor plan contours of the BIM element in order to form irregular walls or openings with rabbeted jambs.

The undisputed advantage of working with the BIM system is to modify the schematic representation of BIM elements which is reflected in its 3D geometry. Nevertheless, the ability to interact remains as well as ability to list elements. After these low-end modifications, 3D solids still remain "intelligent" where in the floor plan it is displayed schematically, keeping interactions and listing the bill of quantities.

\section{Modelling Process}

To illustrate this procedure, the Town Hall renaissance building in Mělník is selected. Survey drawings were delivered in MicroStation DGN files. Survey drawings consisted of the shapes of rooms, elevation dimensions, schema of vault geometry, ceiling heights, vault impost, directions of ceiling beams, and test pits.

DGN files were imported into individual drawings as the base drawings for individual floors. Properties of elements (layers, linetypes, colours, fonts ...) are unified and elements is sorted and cleaned for easy orientation in the base drawings. 


\subsection{Walls and Openings}

It starts with creation of walls. The content of the survey drawing is also copied as the base drawing for creating BIM elements. All layers except walls are switched off. In the base drawing, lines are trimmed to borders, therefore individual areas are closed. Lines of door jambs and window reveals are deleted and unified into intact borders of walls.

The "Polygonal Wall" function is selected to create irregular walls. At the command prompt, "Automatic Geometry Examination" feature is turned on. The BIM elements of the walls are created by clicking inside the closed areas. Because the walls are set same parameters (material, graphics, height), the joined edges are not displayed in connection to the other walls.

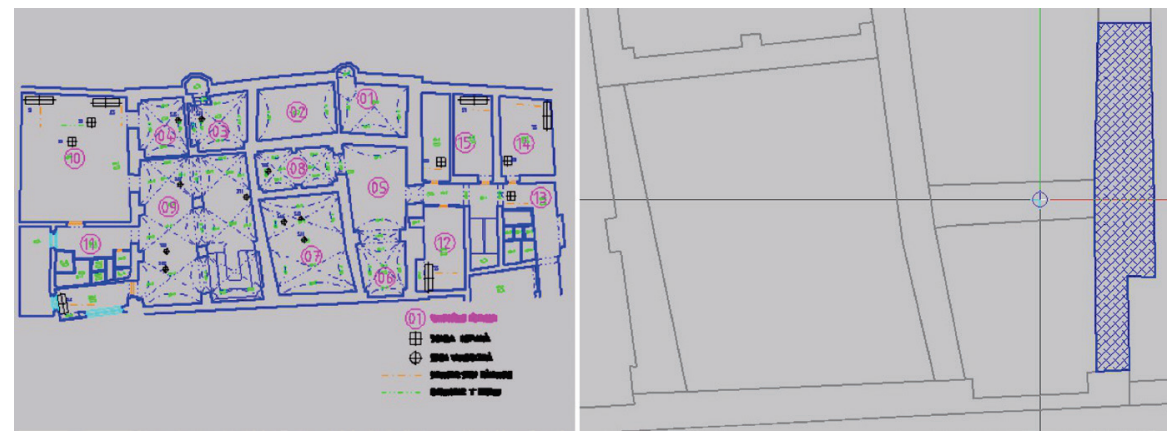

Fig. 2. Lines of survey drawings is used for creating individual BIM "Walls".

After having created the walls, then all layers of base drawing are displayed, so it is possible to snap on the edges when inserting openings. The "Door", "Window", "Niche" functions are used depending on the type of the opening. The location and width of the opening is determined by snapping on the background lines. All walls and openings are now completed.

Door architraves are modelled using 3D solids and stored as parametric objects are referred to "Makro". Also, it is possible to define how the object will be altered when changing the dimension. These objects can be assigned to openings so that they can move, rotate and adapt to the dimensions of the openings, and they are also the billing of quantity depending on various dimensions.

\subsection{Vaults and Ceilings}

After the walls and openings have been created, the vaults are created. For these purposes, the function "Roof Plane" is used which makes a virtual element. The "Roof Plane" function is more general in use. It can be better imagined as a spatial envelope to which BIM elements (walls, openings, ceilings, beams, rooms, roofing...) are adapted. BIM Allplan elements are usually height-related to either the top and/or bottom pairs of "Planes" which is usually represented as the height of the storey. For example, walls are referenced by the lower edge to the lower plane and the upper edge to the upper plane. The ceiling slab is usually referenced to the top plane with the thickness.

This function can be used to define irregular segmental vaults with lunettes over individual rooms. First, an auxiliary room is created outside the room boundary with the same rotation as the room and the average room width. This is because the Allplan "Arc Roof" function can create a segment arc only between strict parallel edges (called "Eaves"). Finally, the parameters of the arc are taken. 
Subsequently, the "Roof Plane" will be created inside the room. The contour will be taken by clicking inside the room with the "Automatic Geometry Examination" feature turned on. The arc from the auxiliary vault is transferred and taken to the setting of the "Mansard Roof" function which is not limited to parallel eaves, and the segment arc will apply to even divergent edges. To create a groin vault or a lunette, the "Dormer" function is used. Same span, arc height and "Eaves" setting for groin vault are used as before ("Eaves" is a substitute name for "Impost" in this case).

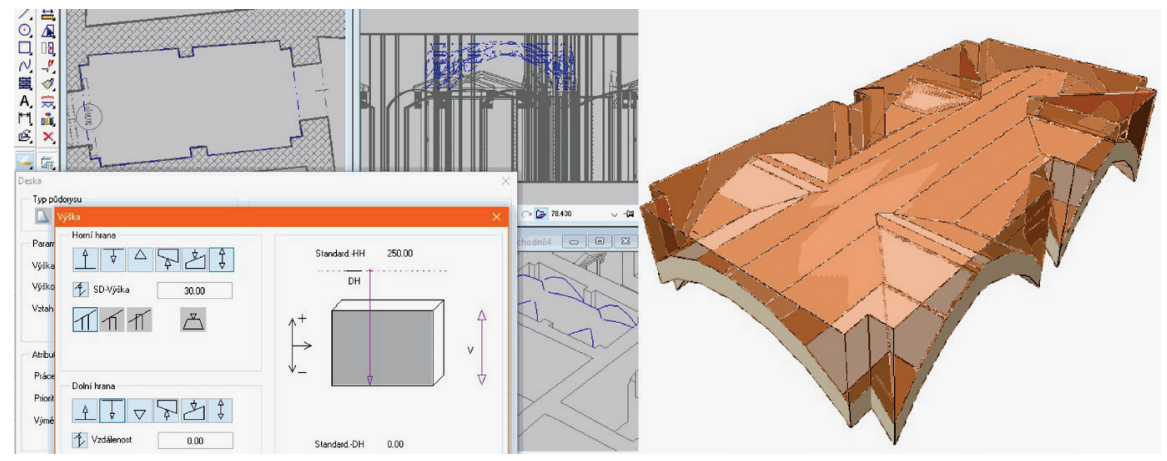

Fig. 3. Creating irregular vaults and settings of BIM elements are referenced to "Planes".

The ceiling plate is created with a bottom edge referenced to the upper "Plane" and the upper edge with a thickness of $30 \mathrm{~cm}$. As the ceiling plate is adapted to the "Roof Plane", a $30 \mathrm{~cm}$ thick vault is created.

The procedures described above are made for the remaining vaults and rooms and all storeys including the attic. The advantage of the virtual spatial envelope is that it adapts not only vaults slabs with infilling, but also to plasters (room surfaces) or to new dividing walls.

The procedure for creating a truss in an attic is not documented here, as it uses procedures and terminology that are identical to the documentation provided with Allplan.

\subsection{Stairs}

Stairs are built with some irregularities. The base drawing is set to display only layers with stair and wall outlines. The "Free Stairs" function is used with "Automatic Geometry Examination" turned on which detects the stair outline. If a mistaken detection occurs, it is advisable to edit lines in the base drawing and trim the walking line from connection to the outline.

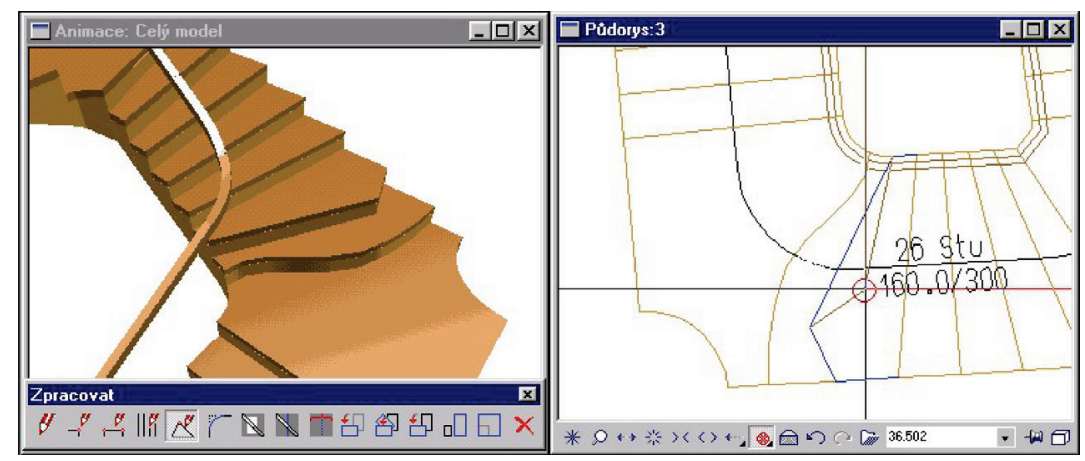

Fig. 4. Modification the schematic representation of BIM elements is reflected in its 3D geometry. 
Subsequently stair parameters are fine-tuned such as the number of steps, the height of the stairs, the insertion of the half-landings, and the degree of rotation. When the steps are rotated, it is enough to rotate only the steps bordering the landings, the other intermediate steps are interpolated.

Each step can be modified with low-end modification functions directly in the floor plan, such as "Add vertex", "Remove vertex", "Modify point" without any disruption of the interaction. All modifications change the $3 \mathrm{D}$ representation and it is listed in the bill of quantity.

\section{Conclusion}

The ability of BIM programs used on historical irregular construction depends on their engine architecture. Therefore, we can conclude that the geometry modeller engine is a more advantageous approach than database or object-oriented engines.

\section{References}

1. Object-oriented design. Available on https://en.wikipedia.org/w/index.php?title=Object-oriented_design\&oldid=79350684

2. H. Nguyen, A. Oloufa, Proceedings of the ICCCBE Conference, 615-620 (1997)

3. Database design. Available on https://en.wikipedia.org/w/index.php?title=Database design\&oldid=800547877

4. G. Lee, J. Jeong, J. Won, C. Cho, S. You, S. Ham, H. Kang, Journal of Computing in Civil Engineering 28 (2), 210-222 (2012)

5. Geometric modeling kernel. Available on https://en.wikipedia.org/w/index.php?title=Geometric_modeling_kernel\&oldid=80512 $\underline{4377}$ 
